\title{
Positionen des Dachverbandes Ärztlicher Diagnostikfächer
}

\section{Wer sind wir?}

Der Dachverband Ärztlicher Diagnostikfächer (DVÄD, ehem. AGMF) ist die Neuorganisation des seit zehn Jahren bestehenden Zusammenschlusses der Berufsverbände der Fachgebiete Radiologie, Pathologie, Nuklearmedizin, Mikrobiologie/Virologie/ Infektionsepidemiologie und der Laboratoriumsmedizin. Wir repräsentieren ärztliche Fachgebiete mit vorwiegend diagnostischem Spezialwissen. Unsere Aufgabe ist es, diese Fachgebiete in ihrer Bedeutung sichtbarer zu machen. Als Querschnittsfächer führen wir diagnostische Leistungen für alle therapeutisch tätigen ÄrztInnen aller Gebiete im haus- und fachärztlichen Bereich und beider Versorgungssektoren durch. Auf unserer Diagnostik basiert die gesamte ärztliche Versorgung. Wir sind grundlegend ärztlich definiert.

\section{Was leisten wir?}

- Die ärztlichen Diagnostikfächer sind systemrelevant. Wir üben eine Schlüsselfunktion in der Vorbereitung, Absicherung, Kontrolle und Monitoring jeglicher Diagnostik und Therapie aus.

- Die ärztlichen Diagnostikfächer sind derzeit der innovativste Bereich der Humanmedizin.

- Wir tragen zur Effizienz und Wirtschaftlichkeit im Gesundheitswesen bei: Durch frühe Erkenntnis individueller Krankheitsfaktoren ermöglichen wir eine schnelle, zielgerichtete Behandlung. Unserer Diagnostik erlaubt, moderne Therapien mit teuren spezifischen Medikamenten bezahlbar einzusetzen. Nicht Diagnostik ist teuer, sondern Therapie.

- Wir arbeiten sektorenübergreifend und ermöglichen PatientInnen so schnitt- stellenfreie Wechsel zwischen den ambulanten und stationären Bereichen.

- Nach den Richtlinien der Bundesärztekammer sowie durch Akkreditierung und Zertifizierung sichern wir die Qualität unserer ärztlichen Arbeit über das gesetzlich vorgeschriebene Maß hinaus.

- Die interdisziplinäre, intersektorale und teamorientierte Ausrichtung unserer Fachgebiete ist impulsgebend für die zukünftige Gestaltung der Patientenversorgung.

\section{Das brauchen wir!}

1. Angemessene Berücksichtigung unserer Fächer in der Ausbildung von ÄrztInnen in Studium, Famulatur und PJ.

2. Weiterbildungsordnungen, die die Kernkompetenzen der ärztlichen Diagnostikfächer schützen und fördern.

3. Sicherung der wirtschaftlichen Grundlagen durch Förderung ärztlicher Leistung in der Diagnostik. Diagnostik ist keine „Finanzierungsreserve“.

4. Beibehaltung der Wahlleistungskette als Ausdruck der notwendigen Zusammenarbeit mit den unmittelbaren krankenversorgenden Fächern.

5. Förderung örtlicher und regionaler Zusammenhänge von Therapeuten und Diagnostikern. Teams sind essenziell für eine höhere Versorgungsqualität. Ihre klare Indikationsbindung senkt die Ausgaben. Überzentralisierung mindert dagegen die Qualität bei höheren Kosten.

6. Eine wohnortnahe und flächendeckende fachärztliche Versorgung mit diagnostischen Leistungen. Die derzeitige zu weiträumige Bedarfsplanung lehnen wir als versorgungsfeindlich ab. KV-Grenzen sind keine Versorgungsgrenzen.

7. Maßnahmen zur Sicherung eines hoch qualifizierten nicht ärztlichen Assistenz- personals in ausreichender Zahl für unseren innovativen Medizinbereich. Dies fordern wir auch in unserer Funktion als großer Arbeitgeber im deutschen Gesundheitswesen.

8. Etablierung einer funktionierenden EDVInfrastruktur zur sicheren Übertragung von Patientendaten und innerärztlicher Kommunikation.

\section{Berufsverband Deutscher Radiologen} e. V.

Bildgebende Diagnostik ist unverzichtbarer Bestandteil der Patientenversorgung im Krankenhaus und in der ambulanten Versorgung.

\section{Bundesverband Deutscher Pathologen e. V.}

Der Pathologe ist Arzt in der Krebsdiagnostik. Neue molekulare Methoden machen individuelle Therapien möglich und bezahlbar.

Berufsverband Deutscher Nuklearmediziner e. $\mathbf{V}$.

Modernste Diagnostik und Therapie mit geringsten Risiken für die Patienten.

Berufsverband Deutscher Laborärzte e. V. Laborärztliche Diagnostik begleitet durch das ganze Leben.

Berufsverband der Ärzte für Mikrobiologie, Virologie und Infektionsepidemiologie e. $\mathbf{V}$.

Aktiv in Labordiagnostik, Infektionsprävention und Infektionsepidemiologie, Krankenhaushygiene sowie infektionsmedizinischer Forschung

Geschäftsstelle:

Robert-Koch-Platz 9, 10115 Berlin

E-Mail: info@DVÄD.de

www.DVÄD.de 